\section{Obesity-related Adipokines and Shoulder Osteoarthritis}

\section{To the Editor:}

It is increasingly understood that the association between obesity and osteoarthritis (OA) is partially mediated by a systemic, inflammatory effect of adipokines such as leptin (LEP), adiponectin (ADIPO), and resistin. The association of obesity and OA of non-weight-bearing joints, such as the hand, lends further support to this hypothesis ${ }^{1,2}$. LEP and resistin have been suggested to have a strong proinflammatory influence ${ }^{3,4}$, while the role of ADIPO in a knee joint is unclear, as some have shown an antiinflammatory effect ${ }^{5}$ and others suggest a proinflammatory effect ${ }^{6}$. Similarly, the ADIPO receptors have been identified in the knee joint only ${ }^{6}$.

No study has examined the epidemiologic relationship between obesity and shoulder OA or identified these obesity hormones in the shoulder joint. The objective of our study was to determine if the adipokines LEP, ADIPO, and resistin are present in the shoulder synovial fluid (SF) of patients with $\mathrm{OA}$ and to examine the relationships of these hormones to measures of body habitus.

We recruited patients awaiting elective arthroscopic rotator cuff repair or shoulder replacement surgery to participate, between 2009 and 2011. We excluded patients with a history of previous shoulder surgery, previous steroid injection, posttraumatic arthritis, or a history of inflammatory arthropathy. Patients undergoing shoulder replacement surgery for a diagnosis of OA were used as the source for articular chondrocytes. The study protocol was approved by the Human Subject Review Committee.

Demographic data, including body mass index (BMI) and waist circumference (WC), were collected. Our study cohort had at least 1 chondral surface with Grade 3 or Grade 4 damage as determined by the Outerbridge classification ${ }^{7}$.

$\mathrm{SF}$ and venous blood samples were collected and stored at $-80^{\circ} \mathrm{C}$; protease inhibitors were added, at the time of shoulder surgery, under sterile conditions. Milliplex ${ }^{\mathrm{TM}}$ MAP kits (Millipore, Natick, MA, USA) were used to detect levels in triplicate of relevant adipokines and inflammatory cytokines. All samples were read on the BioPlex 200 reader.

Performance characteristics for the immunoassays were determined with the intraassay coefficient of variation for each analyte.

Chondrocyte isolation. Cartilage was immediately placed into transport media and kept at $4{ }^{\circ} \mathrm{C}$ until processed. Briefly, cartilage was washed in phosphate buffered saline and transferred to a culture dish containing DMEM supplemented with $10 \%$ fetal bovine serum (FBS) and hyaluronidase. Samples were incubated at $37^{\circ} \mathrm{C}$ overnight and the next day were filtered, centrifuged, resuspended, counted, and plated.

Cell culture. Human liver HepG2 cells, human embryonic kidney HEK293 cells, mouse fibroblast $\mathrm{C} 3 \mathrm{H} 10 \mathrm{~T} 1 / 2$, and mouse prechondrocytic ATDC5 cells were grown in DMEM supplemented with 10\% FBS. ATDC5 and C3H10T1/2 cells were induced to differentiate into chondrocytic cells by the addition of insulin and BMP2, respectively. Samples were passaged through a needle and then centrifuged. Supernatants were collected and protein concentration determined.

Western blot analysis. Samples were run on SDS-polyacrylamide gels and blots were probed with antibodies directed against AdipoR1, AdipoR2, and ObRb.

Immunofluorescence microscopy. Chondrocytes were grown in 12-well Falcon dishes on 18-mm glass coverslips coated with $25 \mu \mathrm{g} / \mathrm{ml}$ poly-L-lysine. Cells were fixed and permeabilized and then incubated in $1 \%$ bovine serum albumin, then left overnight at $4^{\circ} \mathrm{C}$ with AdipoR1, AdipoR2, ObRb, collagen 11, sarcomeric actin, or collagen 1 primary antibodies. All fluorescence images were acquired using a Leica DMI 6000B fluorescence microscope.

Continuous data were compared between groups with the Kruskal-Wallis test, while correlations were determined using nonparametric Spearman coefficients.

Separate linear regression models were built to evaluate the relationship between body habitus (BMI and WC) and the 3 dependent variables: levels of SF LEP, SF ADIPO, and SF resistin. The models were then adjusted for age and sex.

A sample size of 35 patients was chosen for this study, anticipating 4 independent variables in the regression model, thus allowing for $>8$ outcomes per covariate. All statistical analysis was performed with SPSS Version 13.0 (SPSS, Chicago, IL, USA).

In our sample of 35 patients, there were 15 men (43\%), with mean age of 63.0 years (SD 10.1) and mean BMI of $28.3 \mathrm{~kg} / \mathrm{m}^{2}$ (SD 5.4).

Statistically significant correlations were found between SF and serum adipokine levels, that is, LEP $0.39(\mathrm{p}=0.02)$; ADIPO $0.47(\mathrm{p}=0.01)$; and resistin $0.69(\mathrm{p}<0.001)$. Regression modeling showed that female sex and a greater BMI were predictors of higher level of SF LEP, while only a greater BMI predicted a lower level of SF ADIPO, independent of age and WC $(\mathrm{p}<0.05)$. For the model predicting SF resistin levels, no variables reached statistical significance.

The most prevalent receptor detected was the AdipoR1 receptor, which demonstrated a detectable band when as little as $2 \mu \mathrm{g}$ of lysate was run on the gel (Figure 1). Both the AdipoR2 and leptin receptor (ObRb) demonstrated lower levels of expression and were detectable only when $100 \mu \mathrm{g}$ of cell extract was loaded. Immunofluorescence imaging on a confocal microscope revealed a similar pattern of diffuse expression throughout the chondrocytes (Figure 2). Intensity of staining was also found to be comparable across all 3 forms of receptors.

We found that the shoulder joint contained adipokines at levels that correlated with BMI. Further, we identified the LEP ObRb and ADIPO1 and $\mathrm{ADIPO} 2$ receptors on the surface of chondrocytes of late-stage OA shoulders. These findings strengthen the hypothesis of the metabolic pathways of obesity and OA.

Our work is the first to identify that fat-derived hormones are present in OA shoulder joints, whereas other research examining adipokines and $\mathrm{OA}$ of non-weight-bearing joints is limited. One group has shown that greater serum ADIPO may be associated with a decreased risk of progression of radiographic hand $\mathrm{OA}^{8}$, while another showed that greater serum ADIPO is associated with more advanced hand $\mathrm{OA}^{9}$. One final cross-sectional study found that elevated serum resistin was associated with an increased severity of prevalent hand OA, whereas serum ADIPO had no relationship ${ }^{10}$

A limitation of our study is the cross-sectional design, and thus we cannot assume that adipokines observed within the shoulder joint contributed to the OA in our patients.

We successfully identified fat-derived hormones and the corresponding receptors within OA shoulder joints.

RAJIV GANDHI, MD, MS, FRCSC, Assistant Professor, Division of Orthopedic Surgery, University of Toronto; MARK TAKAHASHI, PhD Research Scientist, Division of Orthopedic Surgery, University of Toronto, University Health Network Microarray Centre; RANDY RIZEK, MD, Orthopedic Resident; OMAR DESSOUKI, MD, Orthopedic Resident; NIZAR N. MAHOMED, MD, ScD, Professor, Division of Orthopedic Surgery, University of Toronto.

Address correspondence to Dr. R. Gandhi, Toronto Western Hospital, East Wing 1-439, 399 Bathurst Street, Toronto, Ontario M5T 2S8, Canada.E-mail: rajiv.gandhi@uhn.on.ca

\section{REFERENCES}

1. Grotle M, Hagen KB, Natvig B, Dahl FA, Kvien TK. Obesity and osteoarthritis in knee, hip, and/or hand: An epidemiological study in the general population with 10 years follow-up. BMC Musculoskelet Disord 2008;9:132-6.

2. Gandhi R, Takahashi M, Syed K, Davey JR, Mahomed NN. The relationship between body habitus and leptin in a knee osteoarthritis population. J Orthop Res 2010;28:329-33.

3. Pallu S, Francin PJ, Guillaume C, Gegout-Pottie P, Netter P, Mainard D, et al. Obesity affects the chondrocytes responsiveness to leptin in patients with osteoarthritis. Arthritis Res Ther 


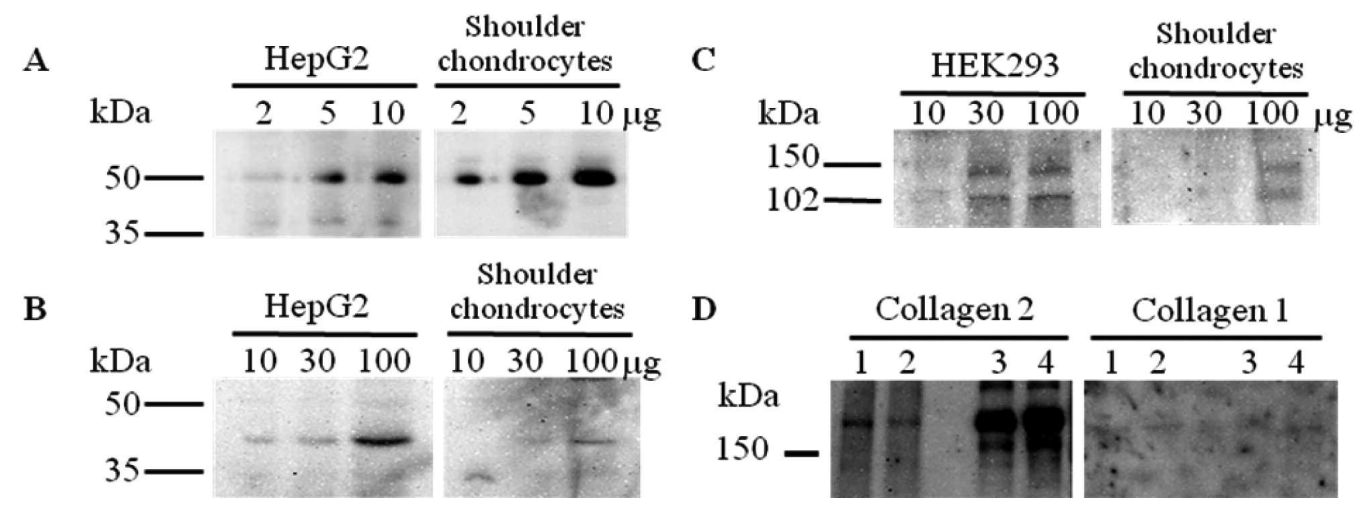

Figure 1. Expression of adiponectin and leptin receptors in shoulder cartilage. Total cell lysates extracted from shoulder cartilage chondrocytes, human hepatic (HepG2) and embryonic kidney (HEK293) cell lines were run on denaturing gels and quantified by Western blotting. Different quantities of cell lysates were loaded: (A) $2 \mu \mathrm{g}$, (B) $5 \mu \mathrm{g}$, and (C) $10 \mu \mathrm{g}$ for the adiponectin type 1 receptor; and (D1) $10 \mu \mathrm{g}$, (D2) $30 \mu \mathrm{g}$, and (D3) $100 \mu \mathrm{g}$ for the adiponectin type 2 and leptin receptors.

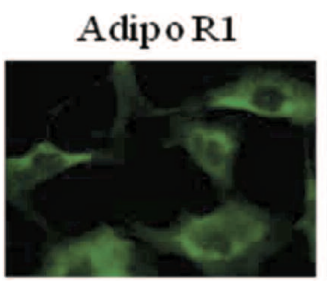

Adipo R2

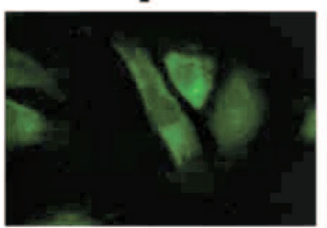

ObRb

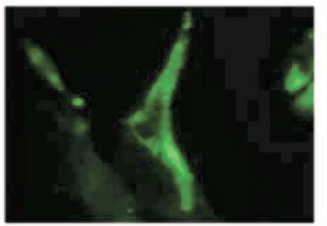

S-actin
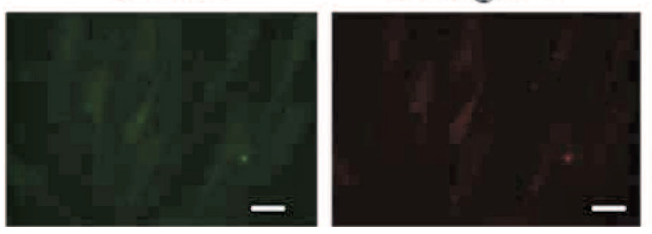

DAPI

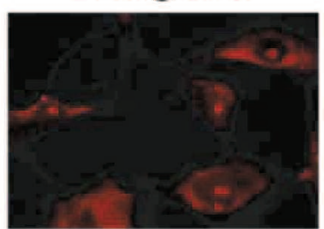

Collagen 11

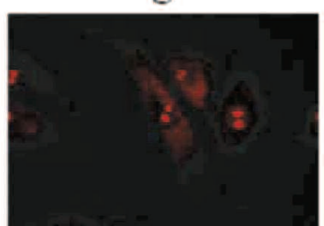

Collagen 11

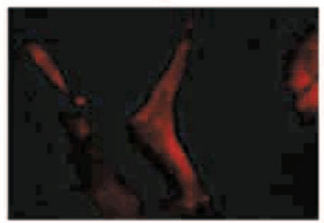

Collagen 1

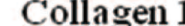

collagen 11 and the nuclear stain DAPI. Scale bars represent $20 \mu \mathrm{m}$.

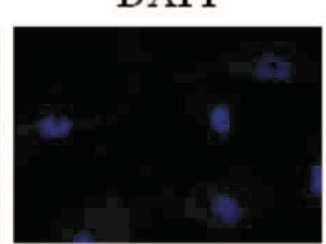

DAPI

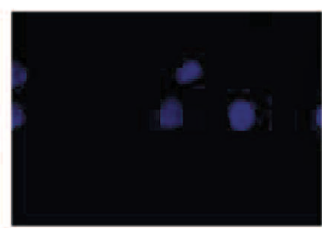

DAPI

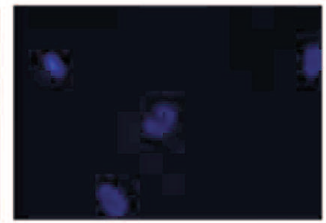

DAPI

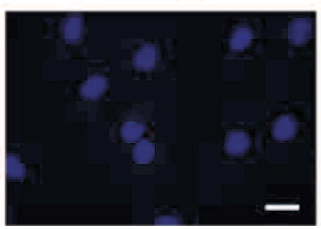

Overlay

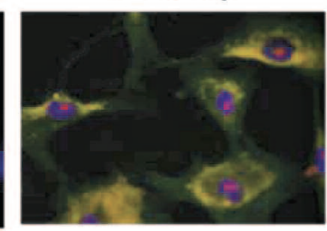

Overlay

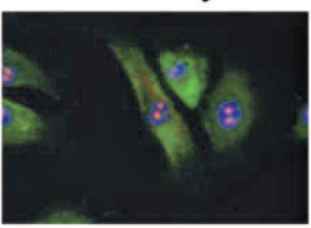

Overlay

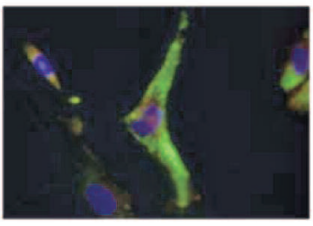

Overlay

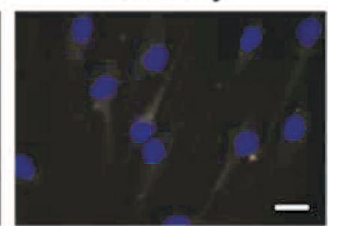

2010;12:R112.

4. Simopoulou T, Malizos KN, Iliopoulos D, Stefanou N, Papatheodorou L, Ioannou M, et al. Differential expression of leptin and leptin's receptor isoform (Ob-Rb) mRNA between advanced and minimally affected osteoarthritic cartilage; effect on cartilage metabolism. Osteoarthritis Cartilage 2007;15:872-83.

5. Chen TH, Chen L, Hsieh MS, Chang CP, Chou DT, Tsai SH.

Evidence for a protective role for adiponectin in osteoarthritis.

Biochim Biophys Acta 2006;1762:711-8.

6. Lago R, Gomez R, Otero M, Lago F, Gallego R, Diequez C, et al. 
A new player in cartilage homeostatsis: Adiponectin induces nitric oxide synthase type II and pro-inflammatory cytokines in chondrocytes. Osteoarthritis Cartilage 2008;16:1101-9.

7. Cameron M, Briggs K, Steadman J. Reproducibility and reliability of the Outerbridge classification for grading chondral lesions of the knee arthroscopically. Am J Sports Med 2003;31:83-6.

8. Yusuf E, Ioan-Facsinay A, Bijsterbosch J, Klein-Wieringa I, Kwekkeboom J, Slagboom PE, et al. Association between leptin, adiponectin and resistin and long-term progression of hand osteoarthritis. Ann Rheum Dis 2011;70:1282-4.
9. Filková M, Lisková M, Hulejová H, Haluzik M, Gatterová J, Pavelková A, et al. Increased serum adiponectin levels in female patients with erosive compared with non-erosive osteoarthritis. Ann Rheum Dis 2009;68:295-6.

10. Choe JY, Bae J, Jung HY, Park SH, Lee HJ, Kim SK. Serum resistin level is associated with radiographic changes in hand osteoarthritis: Cross-sectional study. Joint Bone Spine 2012;79:160-5.

J Rheumatol 2012;39:10; doi:10.3899/jrheum.111339 